\title{
Effectiveness and Feasibility of Complementary Lung-RADS Version I.I in Risk Stratification for PGGN in LDCT Lung Cancer Screening in a Chinese Population
}

This article was published in the following Dove Press journal:

Cancer Management and Research

\section{Qingcheng Meng $\mathbb{D}^{1, *}$ \\ Pengfei Ren $\mathbb{D}^{2, *}$ \\ Pengrui Gao (D) \\ Xinmin Dou' \\ Xuejun Chen (DD' \\ Lanwei Guo $\mathbb{D B}^{3}$ \\ Yongping Song ${ }^{4}$ \\ 'Department of Radiology, The Affiliated Cancer Hospital of Zhengzhou University, Zhengzhou, People's Republic of China; ${ }^{2}$ Department of Molecular Pathology, The Affiliated Cancer Hospital of Zhengzhou University, Zhengzhou, People's Republic of China; ${ }^{3}$ Department of Cancer Prevention Office, The Affiliated Cancer Hospital of Zhengzhou University, Zhengzhou, People's Republic of China; ${ }^{4}$ Department of Hematology, \\ The Affiliated Cancer Hospital of \\ Zhengzhou University, Zhengzhou, \\ People's Republic of China}

*These authors contributed equally to this work

\begin{abstract}
Purpose: To evaluate the effectiveness of using a modified lung imaging reporting and data system (Lung-RADS) for risk stratification of pure ground-glass nodules (pGGNs) in lowdose computed tomography (LDCT) for lung cancer (LC) screenings in China.

Patients and Methods: Eight subjects with nine pGGNs originating from a Cancer Screening Program were enrolled as training set and 32 asymptomatic subjects with 35 pGGNs were selected as validation set from November 2013 to October 2018. The complementary Lung-RADS categories were set based on the GGN-vessel relationship (GVR). The correlations between GGN-vessel relationships and pathology were evaluated, and the diagnostic value of complementary Lung-RADS version 1.1 in discriminating malignant pGGNs were analyzed.
\end{abstract}

Results: The inter-reader agreements for Lung-RADS 1.1 (intraclass correlation coefficient $(\mathrm{ICC}=0.999)$ and complementary Lung-RADS $1.1(\mathrm{ICC}=0.971)$ displayed good reliability. The combined incidence of invasive adenocarcinoma in type III and IV was more than that of benign and preinvasive diseases ( $30 \%$ vs $75 \%, P=0.013)$. Type II GVR between two benign $(66.7 \%)$, seven preinvasive (53.8\%), and six invasive (21.4\%) GGN cases was statistically significant $\left(\chi^{2}=5.415, P=0.019\right)$. GGN pathological groups and GVR had a significant correlation $(\mathrm{r}=0.584$, $P=0.00$ ). Compared to Lung-RADS 1.1, complementary Lung-RADS 1.1 had better performance in the training set, with its sensitivity increased from $33.3 \%$ to $88.9 \%$, accuracy increased from $44.4 \%$ to $88.9 \%$, false-negative proportion (FNP) decreased from $66.7 \%$ to $11.1 \%$, and the sensitivity to predict malignant nodules increased from $13.8 \%$ to $93.1 \%$, accuracy increased from $28.6 \%$ to $80.0 \%$, and FNP decreased from $86.2 \%$ to $6.9 \%$ in validation set. The detection rate of preinvasive disease and adenocarcinoma was increased from $12.5 \%$ to $90.6 \%$ and that of missed diagnosis decreased from $87.5 \%$ to $9.4 \%$ in the validation set, $P=0.004$.

Conclusion: Complementary Lung-RADS 1.1 is superior to Lung-RADS 1.1 and would be beneficial for LC screening of LDCT in China.

Keywords: low-dose computed tomography, lung neoplasms, lung-RADS, mass screening, ground-glass nodules

\section{Introduction}

Early detection and treatment of lung cancer through screening is a promising strategy for improving the detection rate of early lung cancer (LC) and reducing the associated mortality. ${ }^{1}$ LC screening using chest low-dose computed tomography
Department of Hematology, The

Affiliated Cancer Hospital of Zhengzhou

University, Zhengzhou 450008, People's

Republic of China

$\mathrm{Tel} / \mathrm{Fax}+86$ 37I 65587016

Email songyongping00I@I26.com 
(LDCT) is recommended for high-risk individuals, ${ }^{2}$ lung imaging reporting and data system (Lung-RADS) screening interpretation, proposed by the American College of Radiology $(\mathrm{ACR})^{3}$ for LDCT risk stratification, has been used to reduce the false-positive rate with only a small corresponding decrease in sensitivity. ${ }^{4,5}$ Due to the popularity of low-dose, multi-detector spiral computed tomography (CT) in LC screening, ${ }^{6}$ the number of ground-glass nodules (GGNs) or focal ground-glass opacities (fGGOs) detected has increased dramatically. GGN lesion is defined as hazily increased attenuation in the lung with the preservation of intact bronchial and vascular structures, as observed in lung CT imaging. GGNs can be further classified as either mixed ground-glass nodules (mGGNs) or pure ground-glass nodules (pGGNs). The disease spectrum of GGNs includes neoplasms such as pulmonary adenocarcinoma, atypical adenomatous hyperplasia (AAH), and adenocarcinoma in situ (AIS), or benign diseases such as focal fibrosis, inflammation, or alveolar hemorrhages. Screen-detected cancer cases that appear as pGGN and would have been missed or underdiagnosed by LungRADS 1.1, were either smaller than $6 \mathrm{~mm}$ or were GGNs $<30 \mathrm{~mm}\left(14,137.2 \mathrm{~mm}^{3}\right)$. Moreover, a long period of follow-ups recommended by Lung-RADS version 1.1 increases the use of medical resources, causes additional radiation exposure, and leads to patient anxiety. ${ }^{7,8}$ Furthermore, Lung-RADS was designed to be used in the United States where screening programs target high-risk smokers. The diagnostic performance of Lung-RADS in populations with a high prevalence of non-smoking associated LC is unclear, such as in China, Korea, and Japan. ${ }^{9}$ Given the significant differences of LC demographics in Asia and the predominance of subsolid nodules (SSNs) and adenocarcinoma spectrum lesions, the criteria for LC screening and the associated nodule classification and reporting systems requires appropriate adjustments, especially for countries such as China due to its severe air pollution. It is important to provide a personalized harm/ benefit assessment of LDCT LC screening to make informed screening decisions, rather than uniformly recommending or withholding a recommendation for eligible patients. ${ }^{10}$ Hence, we modified Lung-RADS 1.1 for use in the clinical practice. The aim of our pilot study was to evaluate the effectiveness and feasibility of the modified Lung-RADS 1.1 for pGGNs risk stratification in a large number of screening participants.

\section{Materials and Methods}

\section{Study Population}

This retrospective single-center study was approved by the institutional review board of affiliated cancer hospital of Zhengzhou university, patient consent was waived and all of the patient's data were anonymized or maintained with confidentiality. Eight subjects underwent LDCT with 9 pGGNs originated from Cancer Screening Program in Urban China from November 2013 to October 2018 were also selected as training set, which, included 1 male, 7 females with mean age of 58.6 years \pm 11.3 (range, 42-73 years). A total of 32 asymptomatic subjects with 35 pGGNs were enrolled and grouped as validation set at the corresponding period which included 14 males and 17 females with mean age of 53.1 years \pm 9.6 (range, 42-73 years)., all of whom underwent a routine CT before operation. All lesions were confirmed by postoperative pathology. The inclusion criteria were as follows: 1) physical examination or lung cancer screening subjects with pGGN of lung; 2) those who underwent routine CT or LDCT with a thin slice reconstruction $(1.25 \mathrm{~mm})$ and had their image quality should be assessed; 3 ) those with pGGNs possessing well-circumscribed interface, stable after 6 to 12 months of follow-up and pathologically confirmed; 4) those with no history of tuberculosis or inflammation. The exclusion criteria were pGGNs cases without any pathology, poor image quality, subject suffering from dysfunction/apparent damage of important organs and with respiratory symptoms, such as irritable cough, hemoptysis, and chest pain (Figure 1). The clinical characteristics of pGGNs are summarized in Table 1.

\section{Routine CT and LDCT Scanning}

All the subjects of the training set underwent LDCT scanning using a 16-slice multidetector CT (LightSpeed-16, GE, America) and subjects of validation set underwent a routine CT using multidetector CT analyzer (LightSpeed-16, GE, America; iCT-256, Siemens, Germany) device. The protocol parameters were $120 \mathrm{KVp}$ and $200 \mathrm{mAs}$ for routine CT, 120 $\mathrm{KVp}$ and $30 \mathrm{mAs}$ for LDCT, $512 \times 512$ matrix, field of view $400 \mathrm{~mm} \times 400 \mathrm{~mm}$ or $500 \mathrm{~mm} \times 500 \mathrm{~mm}$, collimation $128 \times$ $0.625 \mathrm{~mm}$ or $16 \times 1.25 \mathrm{~mm}$, rotation $0.5 \mathrm{~s}$, pitch 0.8 or 1.02 , $1.25 \mathrm{~mm}$ section width with a $1.25 \mathrm{~mm}$ reconstruction interval and duration of scan 3s-10s. Unenhanced spiral acquisitions were obtained with a breath-hold from the thoracic inlet to lung bases with images. Images were reconstructed using a standard algorithm. All images were sent to a GE ADW 4.5 

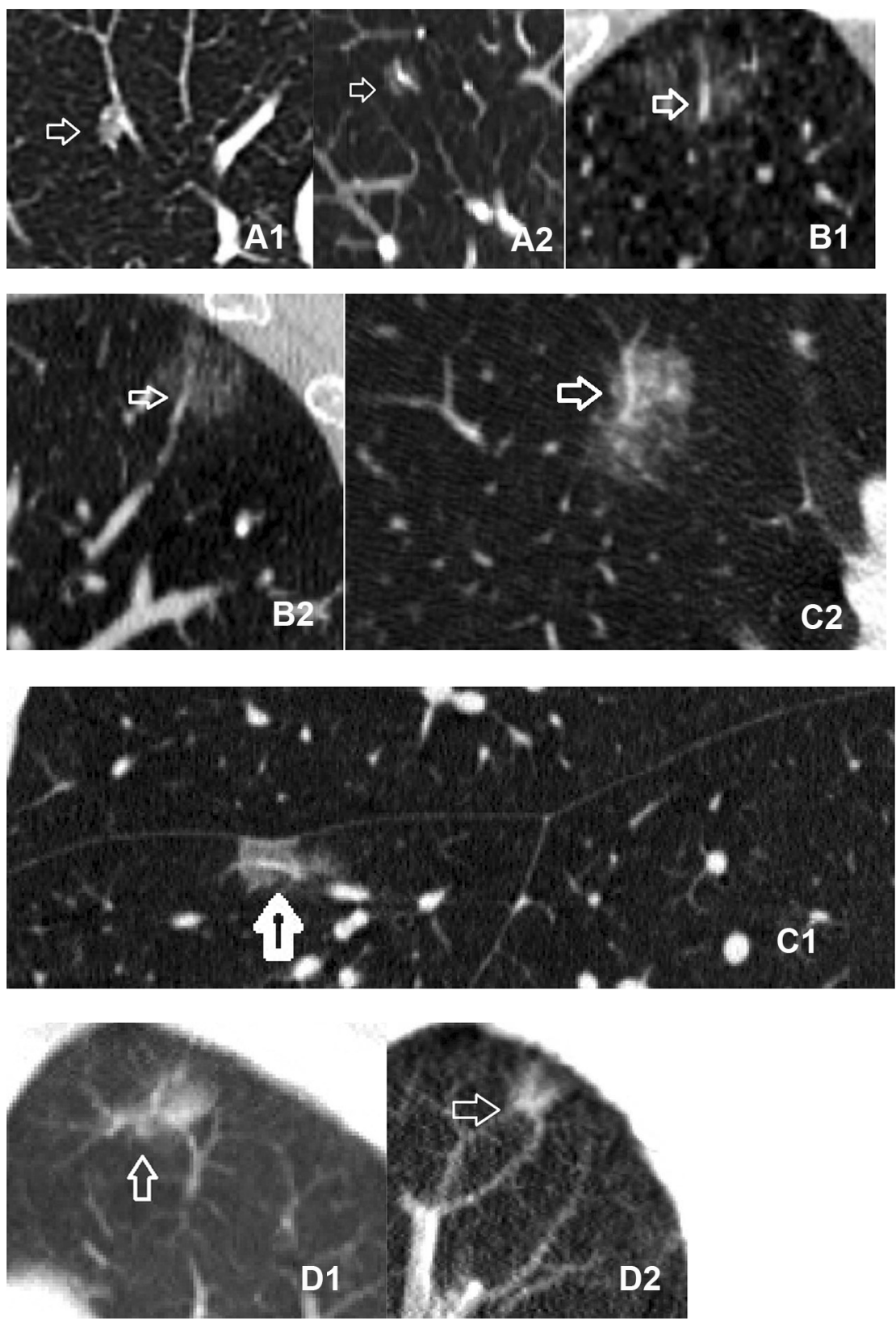

Figure I Flowchart shows risk stratification of nodules by complementary Lung-RADS and Lung-RADS scheme in our study between training set and validation set. Abbreviations: pGGN, pure grand-glass nodule; SN, solid nodule; PSN, part solid nodule; LDCT, low-dose CT; Lung-RADS, lung imaging reporting and data system.

workstation and underwent multiplanar reconstruction (mipPR). All studies were reviewed on a PACS workstation with the window level of -500 to $-700 \mathrm{HU}$ and width of $1400 \mathrm{HU}$.

\section{Management of Lung Nodules and Pathology Diagnosis of LC}

Each pGGN on routine CT or LDCT was independently interpreted by three thoracic radiologists with 7,10 , and
15 years of experience, respectively, who were blinded to the pathological results. For the initial session, all observations were assessed for major and ancillary features in combination according to Lung-RADS V1.1, and then repeated using the complementary Lung-RADS V1.1 algorithm for the second time. In cases of a disagreement on the LI-RADS categories between the three readers, images were re-reviewed together and a consensus categorization was achieved. The nodule size and density were defined accord- 
Table I Clinical Characteristics of Patients Between Training Set and Validation Set (Means \pm Standard Deviations (SD); n (\%))

\begin{tabular}{|c|c|c|c|}
\hline Characteristics & $\begin{array}{l}\text { Training } \\
\text { Set }\end{array}$ & $\begin{array}{l}\text { Validation } \\
\text { Set }\end{array}$ & $P$ \\
\hline \multicolumn{3}{|l|}{ Gender } & 0.221 \\
\hline Male & $\mathrm{I}(\mathrm{I} 2.5)$ & I 4(43.75) & \\
\hline Female & $7(87.5)$ & $18(56.25)$ & \\
\hline Age (y) & $58.6 \pm 11.3$ & $53.1 \pm 9.6$ & 0.383 \\
\hline \multicolumn{3}{|l|}{ Family history of lung cancer } & 0.277 \\
\hline Yes & $\mathrm{I}(12.5)$ & $I(3 . I)$ & \\
\hline No & $7(87.5)$ & $3 I(96.9)$ & \\
\hline \multicolumn{3}{|l|}{ Family history of other cancer } & 1.00 \\
\hline Yes & $0(0.00)$ & $2(6.25)$ & \\
\hline No & $8(100.0)$ & $30(93.75)$ & \\
\hline \multicolumn{3}{|c|}{ Chronic obstructive pulmonary disease } & 1.00 \\
\hline Yes & $\mathrm{I}(12.5)$ & $5(15.6)$ & \\
\hline No & $7(87.5)$ & $27(84.4)$ & \\
\hline Size of pGGNs & $20.0 \pm 9.4$ & $12.5 \pm 6.9$ & 0.010 \\
\hline \multicolumn{3}{|c|}{ Distribution of nodules in patients } & 0.775 \\
\hline One & $8(88.89)$ & $29(90.63)$ & \\
\hline Two & $I(I I . I I)$ & $2(6.25)$ & \\
\hline Three & $0(0.00)$ & $I(3.12)$ & \\
\hline \multicolumn{3}{|l|}{ Lung-RADS I.I } & 0.111 \\
\hline 2 & $6(66.67)$ & $3 I(88.57)$ & \\
\hline 3 & $2(22.22)$ & $\mathrm{I}(2.86)$ & \\
\hline $4 x$ & $\mathrm{I}((\mathrm{II} . \mathrm{II})$ & $3(8.57)$ & \\
\hline \multicolumn{3}{|l|}{ Lung adenocarcinoma spectrum } & 0.649 \\
\hline Benign disease & $0(0.00)$ & $3(8.57)$ & \\
\hline Preinvasive disease(AAH+AIS) & $3(33.33)$ & $10(28.56)$ & \\
\hline $\begin{array}{l}\text { Adenocarcinoma (MIA } \\
\text { +adenocarcinoma) }\end{array}$ & $6(66.66)$ & $22(91.43)$ & \\
\hline \multicolumn{3}{|l|}{ Lung cancer stage } & 0.512 \\
\hline Stage 0 & $3(33.33)$ & $6(17.14)$ & \\
\hline Stage I & $6(66.67)$ & $28(80.0)$ & \\
\hline Stage Ila & & $\mathrm{I}(2.86)$ & \\
\hline
\end{tabular}

Abbreviations: pGGNs, pure ground-glass nodules; Lung-RADS, The ACR Lung Imaging Reporting and Data System; AAH, atypical adenomatous hyperplasia; AIS, adenocarcinoma in situ; MIA, minimally invasive adenocarcinoma.

ing to the Fleischner Society Guidelines. ${ }^{11}$ The GGN-vessel relationships were categorized into four different types according to imaging features: ${ }^{12}$ Type I (pass-by), vessels passing by pGGNs without any detectable supplying branches to the lesions; Type II (pass-through), vessels passing through the lesions without obvious morphological changes in traveling path or size; Type III (distorted/ dilated), vessels within lesions appeared tortuous or rigid without an increase in amount; Type IV (complicated), more complicated vasculature than others described in the aforementioned types within pGGNs, for instance, coexistence of irregular vascular dilation and vascular convergence from multiple supplying vessels (Figure 2). Nodule numbers, sizes, GGN-vessel relationship, and characteristics (pleural indentation, air bronchogram, or vocule sign) were recorded in the structured reports. The average diameters of pGGNs were measured using manual calipers from the largest nodular area for the long- and short-axis diameters in lung window images. Lung-RADS version 1.1 was used to categorize pGGN for risk stratification according to the CT or LDCT images. The pathological diagnosis and categorization of AAH, adenocarcinoma in situ (AIS), microinvasive adenocarcinoma (MIA) and invasive adenocarcinomas (IAC) were made based on the pulmonary adenocarcinoma classification, 2011 edition. ${ }^{13}$ The eighth edition of the TNM Classification for Lung Cancer was used as the histopathology and stage of LC. ${ }^{14}$

\section{Lung-RADS Version I.I and Its Complementary Lung-RADS System}

The Lung-RADS 1.1 of pGGNs were categorized as either 2 (size less than $30 \mathrm{~mm}$ ) or 3 (size equal to or more than $30 \mathrm{~mm}$ ) Table 1. Lung-RADS category 2 (benign appearance) represents as negative screening results, while categories 3 (probably benign) and 4 (suspicious) represent positive results. ${ }^{15}$ Since most of MIA or AIS, even part of invasive adenocarcinoma may appear as pGGNs companied with a well-circumscribed interface, ${ }^{16}$ the majority of pGGNs were categorised as 2 or 3 according to the Lung-RADS version 1.1, which might increase the rate of underdiagnosis. Biological tumor studies have revealed that vasculature remodeling or neoangiogenesis is one of the first occurring events in the early stage of tumor development. Gao et $\mathrm{al}^{12}$ categorized GGN-vessel relationships (GVR) into four types and found that type III and IV of GVR, especially type IV, were strongly correlated with invasive adenocarcinomas. We therefore modified the Lung-RADS version 1.0 according to the type of GVR. First of all, we selected the pGGN with suspicious malignancy based on the $\mathrm{CT}$ morphological features of the well-circumscribed interface, then 6-12months follow-up CT scanning was recommended to exclude inflammation. If the pGGN were stable, maximum intensity projection and multiplanar reconstruction (mipPR) was recommended to 


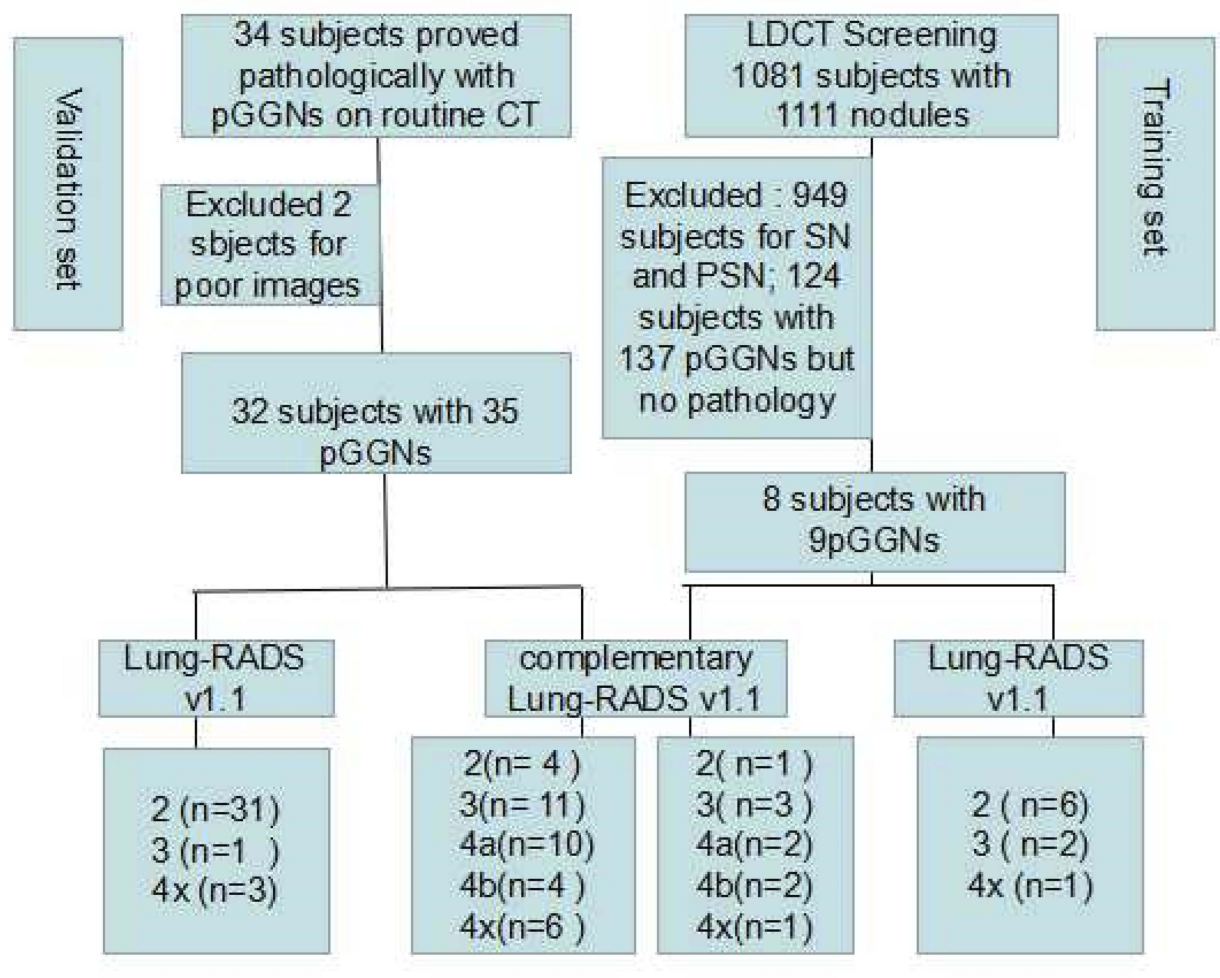

Figure 2 Types of relationship between PGGNs and its vessels: (AI-2): An example of category 2 nodule according to the complementary Lung-RADS I.I. A 34-year-old man with a $0.7 \mathrm{~cm}$ pGGN in right upper lobe (RUL) on the baseline LDCT (AI: axis; A2: coronary). The patient underwent video thoracoscopic subsegment resection of RUL. Further pathologic report demonstrated AIS, Stage 0. (B I-2): A 62-year-old man had a I.5 cm pGGN (complementary Lung-RADS 3) in RUL (BI: coronary; B2: sag). The video thoracoscopic subsegment resection were operated and the pathologic report was MIA, Stage Ia. (CI-2): A 56-year-old woman, there was a pGGN with $2.5 \mathrm{~cm}$ of complementary Lung-RADS 4. The pathologic report was MIA after the video thoracoscopic wedge resection of RML. (DI-2): A 56-year-old woman with multi-nodules in the LUL (DI: coronary; D2: sag), the pathologic report PGGN in anterior segment with complementary Lung-RADS 4 was leptic adenocarcinoma stage Ib.

Abbreviations: pGGN, pure ground-glass nodule; LDCT, low-dose computed tomography; Lung-RADS, the ACR Lung Imaging Reporting and Data System; MIA, minimally Invasive adenocarcinoma; AIS, adenocarcinoma in situ.

Table 2 Summary of Lung-RADS Version I.I of pGGN and Its Complementary Lung-RADS Categories

\begin{tabular}{|c|c|c|}
\hline Category & $\begin{array}{l}\text { Lung-RADS } \\
\text { I.I }\end{array}$ & $\begin{array}{l}\text { Complementary Lung-RADS } \\
\text { I.I }\end{array}$ \\
\hline $\begin{array}{l}2 \\
3 \\
4 a \\
4 b \\
4 x\end{array}$ & $\begin{array}{l}\text { Size }<30 \mathrm{~mm} \\
\text { Size } \geq 30 \mathrm{~mm}\end{array}$ & $\begin{array}{l}\text { Well-circumscribed interface, stable } \\
\text { after } 6 \text { to I } 2 \text { months follow-up } \\
\text { Type I of GVR and Size }<30 \mathrm{~mm} \\
\text { Type I of GVR and Size } \geq 30 \mathrm{~mm} \text {; type } \\
\text { II of GVR } \\
\text { Any size with type III of GVR } \\
\text { Any size with type IV of GVR } \\
\text { Category } 3 \text { or } 4 \text { nodules with } \\
\text { additional features or imaging } \\
\text { findings that increases the suspicion } \\
\text { of malignancy }\end{array}$ \\
\hline
\end{tabular}

Abbreviations: GVR, GGN-vessel relationship; Lung-RADS, lung imaging reporting and data system.

evaluate the supplying blood vessels. We identified the type I of GVR and size $<30 \mathrm{~mm}$ as Lung-RADS 2, type I of GVR and size $\geq 30 \mathrm{~mm}$ or type II of GVR as Lung-RADS 2 , and any sizes with GGN of type III as Lung-RADS 4a and with type IV as Lung-RADS $4 b$, Category 3 or 4 nodules with additional features or imaging findings that increased the suspicion of malignancy were defined as Lung-RADS 4x (Table 2). Category 3 and 4 nodules were defined as positive. The risk stratification of 44 pGGNs in our study was reclassified using complementary Lung-RADS 1.1 (Figure 2).

\section{Statistical Analysis}

Statistical analysis was performed with SPSS 24.0 (SPSS Inc, Chicago, IL) and MedCalc 15.2.2 (MedCalc Software, Ostend, Belgium). Data were reported as mean \pm standard deviation (SD). An independent $t$-test was used to compare quantitative data. Counting data were described by frequency and percentage, and comparison between groups was conducted by Chi-square test. When the expected value was $<1$ or the pretest probability was approximately the same as the test level, Fisher's exact test was used instead. The interreader reliability of Lung-RADS categories between three readers were calculated by intraclass correlation coefficient 
(ICC). Correlation between pathological GGN findings and GVR were examined using the Spearman rank test. The validity and predictive value of complementary Lung-ADS were calculated for sensitivity, accuracy, detection rate, and rate of missed diagnosis, respectively. A $P$-value of less than 0.05 was considered significant.

\section{Results}

\section{Demographics and Clinical}

\section{Characteristics of pGGNs}

The clinical characteristics of 39 study subjects with 44 pGGNs are shown in Table 1. Eight subjects with nine pGGNs in the training set contained one subject with two nodules and seven subjects with one nodule. Six pGGNs were classified as category 2, 2 as category 3, and one as category $4 \mathrm{x}$ and there were three AISs, three MIAs, and three invasive adenocarcinomas according to the Lung adenocarcinoma spectrum. In the validation set, there was one subject with three nodules, one subject with two nodules and 29 subjects with only one nodule. It also had 37 pGGNs classified as category 2,3 as category 3 , and 4 as category $4 \mathrm{x}$ according to the Lung-RADS 1.1. The disease spectrum included three inflammation diseases, 4 AAHs, 6 AISs, 7 MIAs, and 15 invasive adenocarcinomas. Two participants had a family history of LC, two cases had a family history of other cancers and six participants were occupied with chronic obstructive pulmonary diseases. The size of pGGNs between the training set and the validation set was statistically significantly $(P=0.010)$. No statistical significance ( $P \square 0.05$ ) was observed between training set and validation set for the Lung-RADS category, sex, subjects with nodule number, the family history of LC, and other malignant tumors, the history of other pulmonary chronic diseases, lung adenocarcinoma spectrum and its tumor stage, and lung benign spectrum of the subjects.

\section{Inter-Reader Reliability Agreement for PGGNs Category Based on LI-RADS VI.I and Complementary Lung-RADS VI.I}

The Lung-RADS categories of all observations for each reader based on both v1.1 and complementary v1.1 algorithm are shown in Table 3. The interreader agreements for Lung-RADS 1.1 (0.999 [0.986-1.00]) and complementary Lung-RADS 1.1 (0.971 [0.854-0.997]) exhibited good reliability coefficients.

\section{Correlations Between GVR and Pathological Findings}

In order to observe the relationship between types of GGNvessel relationship and its pathology, we divided GGN as the benign disease group ( 3 inflammations), preinvasive group (including 4 AAHs, 9 AISs), and adenocarcinoma group (28 cases) according to the pathologic findings. Of the 44 GGNs, type I, II, III, and IV GVR were observed in 5, 15, 7, and 17 cases, respectively (Figure 2). Compared to the incidence of type III and IV relationships in benign and preinvasive groups (combined type III + IV, $0.0 \%$ and $30 \%$, respectively), the combined incidence of type III (17.9\%) and IV $(57.1 \%)$ relationships in the invasive adenocarcinoma group reached $75.0 \%\left(\chi^{2}=6.117, P=0.013\right)$. The total number of GGNs with type III and IV was 24 , including 3 preinvasive

Table 3 Lung-RADS Categories in Each Reader Based on Version I.I and Complementary Version I.I, and Inter-Reader Reliability Assessment

\begin{tabular}{|c|c|c|c|c|c|c|}
\hline Lung-RADS Version & Category & Reader I & Reader 2 & Reader3 & ICC & $P$ value \\
\hline \multicolumn{5}{|l|}{ Lung-RADS I.I } & $0.999(0.986,1.00)$ & 0.000 \\
\hline & 2 & 36 & 37 & 38 & & \\
\hline & 3 & 3 & 4 & 2 & & \\
\hline & $4 X$ & 5 & 3 & 4 & & \\
\hline \multicolumn{5}{|c|}{ Complementary Lung-RADS I.I } & $0.97 I(0.854,0.997)$ & 0.000 \\
\hline & 2 & 5 & 5 & 4 & & \\
\hline & 3 & 12 & 16 & 14 & & \\
\hline & $4 a$ & 8 & 6 & 7 & & \\
\hline & $4 b$ & 13 & 13 & 13 & & \\
\hline & $4 x$ & 6 & 4 & 6 & & \\
\hline
\end{tabular}

Abbreviations: Lung-RADS, lung imaging reporting and data system; ICC, intraclass correlation coefficient. 
nodules and 21 adenocarcinomic nodules, indicating that the presence of the type III and IV GGN-vessel relationship, especially type IV, strongly suggested a high likelihood of malignancy. Correlation analysis showed that a correlation existed between the GGN pathological groups and the GVR $(\mathrm{r}=0.584, \mathrm{P}=0.00)$. Even though type II GGN-vessel relationship was one of the most common GVR, accounting for $27.3 \%$, seen in two benign $(66.7 \%)$, seven preinvasive $(53.8 \%)$ and six invasive $(21.4 \%)$ GGN cases, but was statistically significant among them $\left(\chi^{2}=5.415, P=0.019\right)$, which may also inclined to benign or preinvasive diseases, shown in Table 4.

\section{Evaluation of the Improved Diagnostic Value of Complementary Lung-RADS in Discriminating Benign and Malignant Pulmonary Nodules}

As shown in Table 5, evaluation of the diagnostic value showed that compared to Lung-RADS 1.1, the sensitivity

Table 4 The Incidence Rate of GGN-Vessel Relationship Types of Three GGN Groups

\begin{tabular}{|c|c|c|c|c|}
\hline \multirow[t]{2}{*}{ Groups } & \multicolumn{4}{|c|}{ GGN-Vessel Relationship } \\
\hline & Type I & Typ II & Type III & Type IV \\
\hline Benign group & $\mathrm{I} *(33.3 \%)^{\#}$ & $2(66.7 \%)$ & $0(0.0 \%)$ & $0(0.0 \%)$ \\
\hline $\begin{array}{l}\text { Preinvasive } \\
\text { group }\end{array}$ & $3(23.1 \%)$ & $7(53.8 \%)$ & $2(15.4 \%)$ & $\mathrm{I}(7.7 \%)$ \\
\hline $\begin{array}{l}\text { Adenocarcinoma } \\
\text { group }\end{array}$ & $\mathrm{I}(3.6 \%)$ & $6(21.4 \%)$ & $5(17.9 \%)$ & $16(57.1 \%)$ \\
\hline
\end{tabular}

Notes: *Case(s) corresponding to particular type and group; "Proportion of particular type in the corresponding group. of complementary Lung-RADS 1.1 prediction for malignant pulmonary nodules in the training set was increased from $33.3 \%$ to $88.9 \%$, the accuracy increased from $33.3 \%$ to $88.9 \%$, and false negative proportion (FNP) decreased from $66.7 \%$ to $11.1 \%$. The sensitivity of complementary Lung-RADS 1.1 prediction for malignant pulmonary nodules in the validation set increased from $13.8 \%$ to $93.1 \%$, accuracy increased from $28.6 \%$ to $80.0 \%$, FNP decreased from $86.2 \%$ to $6.9 \%$. The optimal cut-off value of the two methods was classified as 3 , which meant that nodules categories 3 and above, including categories $4 a, 4 b$, and $4 x$, were defined as positive nodules. Whereas categories below 3, including categories 1 and 2 , were defined as negative nodules. The detection rate of preinvasive disease and adenocarcinoma in the training set was increased from $33.3 \%(12.1,64.6)$ to $88.9 \%(56.5,98.0)$ and the rate of missed diagnosis decreased from $66.7 \%(35.4,87.9)$ to $11.1 \%(2.0,43.5), P=0.050$ (Figure 3$)$. The detection rate of preinvasive disease and adenocarcinoma in the validation set was increased from $12.5 \%(5.0,28.1)$ to $90.6 \%(75.8$, 96.8) and the rate of missed diagnosis decreased from $87.5 \%$ $(71.9,95.0)$ to $9.4 \%(3.2,24.2), P=0.004$ (Figure 4).

Taken together, these results suggested that the complementary Lung-RADS 1.1 proposed in this study is an effective and better screening method than Lung-RADS 1.1.

\section{Discussion}

In this study, we modified the Lung-RADS 1.1 to categorize the pGGNs according to the GGN-vessel relationship. The results suggest that complementary Lung-RADS 1.1 performs better than Lung-RADS 1.1. While revising the complementary Lung-RADS, one of the major

Table 5 Comparison of Diagnostic Value for Benign and Malignant of Lung Nodule with Lung-RADS I.I Or Complementary Lung RADS I.I

\begin{tabular}{|l|l|l|l|l|}
\hline \multirow{2}{*}{} & \multicolumn{2}{l|}{ Training Set } & \multicolumn{2}{l|}{ Validation Set } \\
\cline { 2 - 5 } & Lung-RADS I.I & Complementary Lung-RADS I.I & Lung-RADS I.I & Complementary Lung-RADS I.I \\
\hline TP & 3 & 8 & 4 & 27 \\
FP & 0 & 0 & 0 & 5 \\
FN & 6 & 1 & 25 & 2 \\
TN & 0 & 0 & 6 & 1 \\
Sensitivity, \% (95\% Cl) & $33.3(12.1,64.6)$ & $88.9(56.5,98.0)$ & $13.8(5.5,30.6)$ & $93.1(78.03,98.1)$ \\
FNP,\% (95\% Cl) & $66.7(35.4,87.9)$ & $11.1(2.00,43.5)$ & $86.2(69.4,94.5)$ & $6.9(1.9,22.0)$ \\
PPV, \% (95\% Cl) & $100.0(43.9,100)$ & $100.0(67.6,100)$ & $100.0(51.0,100)$ & $84.4(68.3,94.1)$ \\
NPV, \% (95\% Cl) & $0.0(0,39.0)$ & $0.0(0,79.4)$ & $19.4(9.2,36.3)$ & $33.3(6.2,79.2)$ \\
Accuracy, \% (95\% Cl) & $33.3(12.1,64.6)$ & $88.9(56.5,98.0)$ & $28.6(16.3,45.1)$ & $80.0(64.1,90.0)$ \\
\hline
\end{tabular}

Abbreviations: FN, false negative; FP, false positive; TN, true-negative; TP, true-positive; FNP, false negative proportion; PPV, positive predictive value; NPV, negative predictive value; $95 \% \mathrm{Cl}$, confidence interval. 


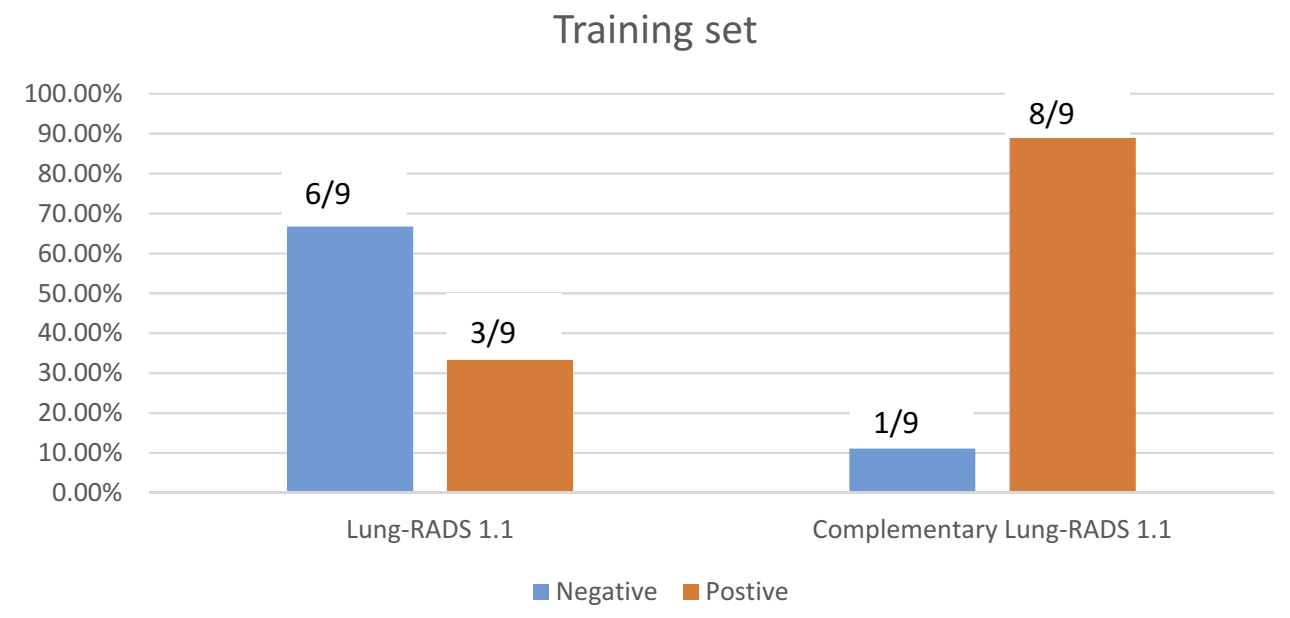

Figure 3 Detection rate or missed diagnosis rate of preinvasive disease and adenocarcinoma in training set with threshold value of Lung-RADS 3 category. The detection rate of preinvasive disease and adenocarcinoma in training set was increased from 33.3\% (I2.I, 64.6) to 88.9\% (56.5, 98.0); as the rate of missed diagnosis decreased from $66.7 \%(35.4,87.9)$ to $11.1 \%(2.0,43.5), P=0.050$.

Abbreviation: Lung-RADS, lung imaging reporting and data system.

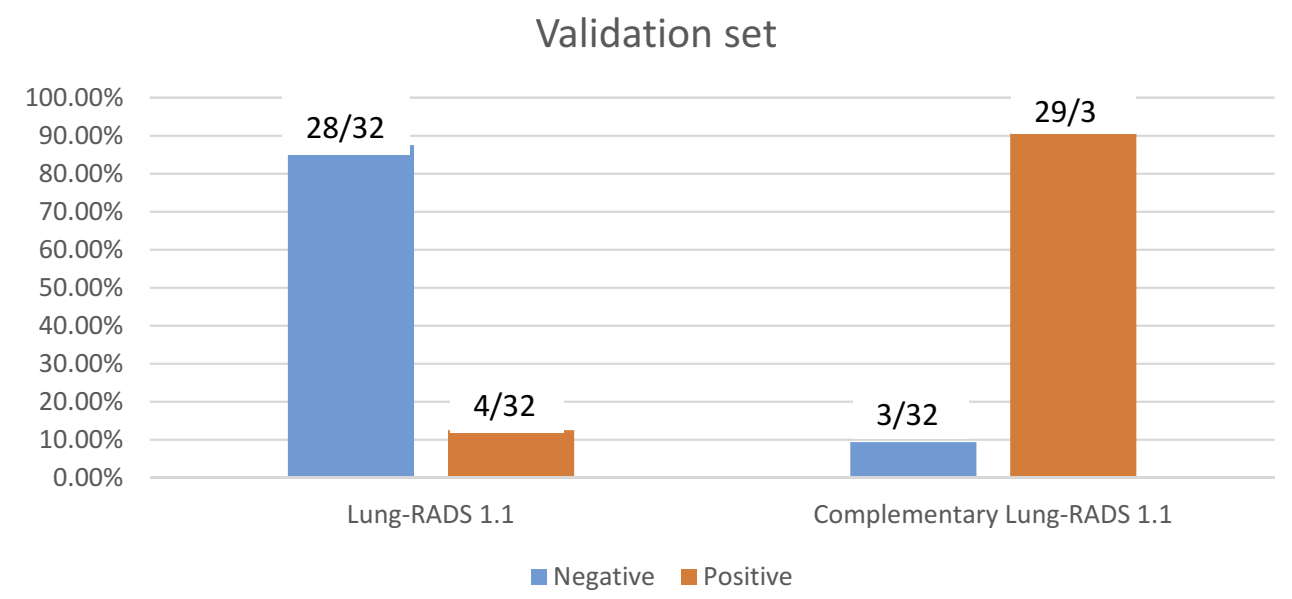

Figure 4 Detection rate or missed diagnosis rate of preinvasive disease and adenocarcinoma in Validation set with threshold value of Lung-RADS 3 category. The detection rate of preinvasive disease and adenocarcinoma in validation set was increased from $12.5 \%(5.0,28.1)$ to $90.6 \%(75.8,96.8)$; as the rate of missed diagnosis decreased from $87.5 \%(71.9,95.0)$ to $9.4 \%(3.2,24.2), P=0.004$

Abbreviation: Lung-RADS, lung imaging reporting and data system.

problems was the relationship correlation between the pathology of pGGN and the GVR, the fundamental mechanism of which was vasculature remodelling or neoangiogenesis occurring in the early stage of tumor development and the tumoral angiogenesis or neovascularization could be the driving factors of vascular abnormalities, as observed in several other studies ${ }^{17,18}$ and appeared as distorted, rigid or concentrated toward the lesions in the CT images. Our results showed that GRV had a better correlation with GGN pathological groups ( $\mathrm{r}=0.584$, $P=0.00$ ), and type III and IV GGN-vessel relationship, especially type IV, strongly suggested a high likelihood of malignancy similar to previous studies. ${ }^{12,19,20}$ First, malignant lesions with greater growth and metabolism require more blood flow and therefore induce the surrounding vessels to broaden. Second, Noguchi's et $\mathrm{al}^{21}$ stated that fibrosis reaction and subsequent tissue contraction was the main mechanism for generating vascular convergence signs (VCS), and with the invasive grade of the tumor increasing, fibrotic foci enlarge to cause vessel contraction. In our study, type II GGN-vessel relationship had a correlation to benign or preinvasive diseases. Gao et $\mathrm{al}^{12}$ also showed that the type II GGN-vessel relationship was common among benign and malignant GGN groups. Based on the above results, we defined the type II relationship of pGGNs as a hallmark of a lesion, 
possessing an independent blood supply, with momentums of continuous and accelerated progression, and caution for potential malignancy.

In our study, the inter-reader agreements for LungRADS categories for v1.1 (0.999 [0.986-1.00]) and complementary v1.1 (0.971 [0.854-0.997]) all displayed good reliability coefficients, which proves the good application of complementary Lung-RADS 1.1 in a clinical setting. Nine pGGNs were selected as the training set from the 137 pGGNs screened from the Cancer Screening Program in China and were followed up for a long time to repeat LDCT screening. ${ }^{22}$ This may have added additional emotional stress and ultimately affected the cost-effectiveness of screening. Persistent pGGNs or mGGNs are often lung adenocarcinoma spectrum lesions and include invasive adenocarcinoma or its precursors. A recent systematic review of the psychological burden of LDCT revealed that LDCT screening might be associated with a short-term psychological burden in participants, ${ }^{7}$ A female NELSON study reported a worse health-related quality of life outcomes among females compared to males. ${ }^{23}$ Our study results showed that compared to the Lung-RADS 1.1, the sensitivity and accuracy of complementary Lung-RADS 1.1 to predict malignant pulmonary nodules both in the training set and the validation set were remarkably increased. The detection rate of preinvasive disease and adenocarcinoma in training set was increased from $33.3 \%$ (3/9) to $88.9 \%$ (8/ $9)$; while the rate of missed diagnosis decreased from $66.7 \%(6 / 9)$ to $11.1 \%(1 / 9)$. This suggests that complementary lung-RADS 1.1 is a better screening method than lung-RADS 1.1 .

Our study did have some limitations. The study is retrospective and single-centered, which may be affected by the bias of design. For example, pGGNs without pathology were excluded, therefore, there was a lack of further confirmed validation of complementary LungRADS 1.1 due to only a few cases of pGGNs being enrolled. The small sample size was the other limitation of this study, especially in the benign group, which may have compromised the diagnostic power. In the end, LDCT screening with modified Lung-RADS 1.1 cannot avoid the risk of overdiagnosis and overtreatment as these pGGNs may be indolent and clinically insignificant resulting in the remainder of the patient's life being subclinic. However, Henschke et al found that nearly $90 \%$ of the diagnosed but untreated stage IA nonsmall cell LC as small as $10 \mathrm{~mm}$ in diameter has a malignant natural course and is fatal if not treated. ${ }^{24}$ Therefore, further evaluation of the effectiveness of complementary Lung-RADS 1.1, with more pGGNs cases, is warranted.

\section{Conclusion}

Complementary Lung-RADS is an effective method for LDCT screening of LC cases in the Chinese population. Its effectiveness should be further verified in a large-scale study.

\section{Acknowledgments}

We would like to thank the Cancer Screening Program in Urban of National Cancer Center in China for its data of LDCT screening of LC made available free. We would also like to thank Pengfei Ren Ph.D. for his statistical contribution. All authors had full access to the data in the study and take full responsibility for the integrity of the data and the accuracy of the data analysis.

\section{Disclosure}

The authors report no conflicts of interest in this work.

\section{References}

1. Aberle DR, Berg CD, Black WC, et al. The National Lung Screening Trial: overview and study design. Radiology. 2011;258:243-253. doi:10.1148/radiol.10091808

2. Aberle DR, Adams AM, Berg CD; National Lung Screening Trial Research Team, et al. Reduced lung-cancer mortality with low-dose computed tomographic screening. N Engl J Med. 2011;365:395-409. doi:10.1056/NEJMoa1102873

3. American College of Radiology. Lung CT screening reporting and data system (Lung-RADSTM). 2014. Available from: http://www.acr.org/ Quality-Safety/Resources/LungRADS. Accessed January 2, 2020.

4. Pinsky PF, Gierada DS, Black W, et al. Performance of lung-RADS in the National Lung Screening Trial: a retrospective assessment. Ann Intern Med. 2015;162(7):485-491. doi:10.7326/M14-2086

5. Wu FZ, Huang YL, Wu CC, et al. Assessment of selection criteria for low-dose lung screening CT among Asian ethnic groups in Taiwan: from mass screening to specific risk-based screening for non-smoker lung cancer. Clin Lung Cancer. 2016;17(5):e45-e56. doi:10.1016/j.cllc.2016.03.004

6. Tammemägi MC, Katki HA, Hocking WG, et al. Selection criteria for lung-cancer screening. $N$ Engl $J$ Med. 2013;368(8):728-736. doi:10.1056/NEJMoa1211776

7. Wu GX, Raz DJ, Brown L, et al. Psychological burden associated with lung cancer screening: a systematic review. Clin Lung Cancer. 2016;17:30053-30055. doi:10.1016/j.cllc.2016.03.007

8. Taghizadeh N, Tremblay A, Cressman S, et al. Health-related quality of life and anxiety in the PAN-CAN lung cancer screening cohort. BMJ Open. 2019;9(1):e024719. doi:10.1136/bmjopen-2018-024719

9. Detterbeck FC, Franklin WA, Nicholson AG, et al. The IASLC lung cancer staging project: background data and proposed criteria to distinguish separate primary lung cancers from metastatic foci in patients with two lung tumors in the forthcoming eighth edition of the TNM classification for lung cancer. $J$ Thorac Oncol. 2016;11(5):651-665. doi:10.1016/j.jtho.2016.01.025 
10. Caverly TJ, Cao P, Hayward RA, Meza R. Identifying patients for whom lung cancer screening is preference-sensitive: a microsimulation study. Ann Intern Med. 2018;169(1):1-9. doi:10.7326/M17-2561

11. Naidich DP, Bankier AA, MacMahon H, et al. Recommendations for the management of subsolid pulmonary nodules detected at CT: a statement from the Fleischner Society. Radiology. 2013;266 (1):304-317. doi:10.1148/radiol.12120628

12. Gao F, Li M, Ge X, et al. Multi-detector spiral CT study of the relationships between pulmonary ground-glass nodules and blood vessels. Eur Radiol. 2013;23(12):3271-3277. doi:10.1007/s00330013-2954-3

13. Travis WD, Brambilla E, Noguchi $\mathrm{M}$, et al. International association for the study of lung cancer/American thoracic society/European respiratory society international multidisciplinary classification of lung adenocarcinoma. $J$ Thorac Oncol. 2011;6:244-285. doi:10.1097/JTO.0b013e318206a221

14. Detterbeck FC, Boffa DJ, Kim AW, et al. The eighth edition lung cancer stage classification. Chest. 2017;151(1):193-203. doi:10.1016/ j.chest.2016.10.010

15. Lee JW, Kim HY, Goo JM, et al. Radiological report of pilot study for the korean lung cancer screening (K-LUCAS) project: feasibility of implementing lung imaging reporting and data system. Korean J Radio. 2018;19:803-808.

16. Meng QC, Gao PR, Ren PF, Song YP, Li HL. Early diagnosis of subtype in early clinical stage lung adenocarcinoma by using an autoantibody panel and computed tomography. Zhonghua Yi Xue Za Zhi. 2019;99 (3):204-208. doi:10.3760/cma.j.issn.0376-2491.2019.03.010

17. Folkman J. Angiogenesis in cancer, vascular, rheumatoid and other disease. Nat Med. 1995;1(1):27-31. doi:10.1038/nm0195-27
18. Fontanini G, Vignati S, Boldrini L, et al. Vascular endothelial growth factor is associated with neovascularization and influences progression of non-small cell lung carcinoma. Clin Cancer Res. 1997;3 (6):861-865.

19. Gao F, Sun Y, Zhang G, Zheng X, Li M, Hua Y. CT characterization of different pathological types of subcentimeter pulmonary ground-glass nodular lesions. Br J Radiol. 2019;92(1094):20180204. doi:10.1259/ bjr.20180204

20. Wang X, Wang L, Zhang W, Zhao H, Li F. Can we differentiate minimally invasive adenocarcinoma and non-invasive neoplasms based on high-resolution computed tomography features of pure ground glass nodules? PLoS One. 2017;12(7):e0180502. doi:10.1371/journal.pone.0180502

21. Noguchi M, Morikawa A, Kawasaki M, et al. Small adenocarcinoma of the lung. Histologic characteristics and prognosis. Cancer. 1995;75 (12):2844-2852. doi:10.1002/1097-0142(19950615)75:12<2844::aidcncr2820751209>3.0.co;2-\#

22. Kim HY, Jung KW, Lim KY, et al. Lung cancer screening with low-dose CT in female never smokers: retrospective cohort study with long-term national data. follow-up. Res Treat. 2018;50 (3):748-756. doi: $10.4143 /$ crt.2017.312

23. van den Bergh KA, Essink-Bot ML, Borsboom GJ, et al. Short-term health-related quality of life consequences in a lung cancer CT screening trial (NELSON). Br J Cancer. 2010;102:27-34. doi:10.1038/sj. bjc. 6605459

24. Henschke CI, Wisnivesky JP, Yankelevitz DF, Miettine OS. Small stage I cancers of the lung: genuineness and curability. Lung Cancer. 2003;39(3):327-330. doi:10.1016/S0169-5002(02)00503-2

\section{Publish your work in this journal}

Cancer Management and Research is an international, peer-reviewed open access journal focusing on cancer research and the optimal use of preventative and integrated treatment interventions to achieve improved outcomes, enhanced survival and quality of life for the cancer patient.
The manuscript management system is completely online and includes a very quick and fair peer-review system, which is all easy to use Visit http://www.dovepress.com/testimonials.php to read real quotes from published authors. 